\title{
Clinical and treatment aspects in chronic hypertrophic rhinitis in children
}

\author{
${ }^{\star}$ Emilia Gheorghian, Mihail Maniuc, Polina Ababii, Lucian Danilov
}

\author{
Department of Otorhinolaryngology \\ Nicolae Testemitanu State University of Medicine and Pharmacy, Chisinau, the Republic of Moldova
}

Authors' ORCID iDs, academic degrees and contributions are available at the end of the article

${ }^{\star}$ Corresponding author - Emilia Gheorghian, e-mail: emiliagheorghian@gmail.com

Manuscript received May 12, 2021; revised manuscript July 12, 2021; published online September 10, 2021

\begin{abstract}
Background: Chronic hypertrophic rhinitis is a common problem in childhood and adolescence and has a negative impact on both physical, social and psychological well-being. Chronic hypertrophic rhinitis in the prevalence of children is $20-55 \%$, and in the last 5 years this index is increasing. Material and methods: The study included 65 patients, aged 5 to 18 years, being hospitalized in the ENT department of the Emilian Cotaga Clinic of the Institute of Mother and Child, Chisinau, diagnosed with rhinitis complicated chronic hypertrophic over the years January 2018 - December 2020 . The study was conducted by retrospective analysis of complicated data from clinical observation sheets and documents accompanying the data. Treatment methods studied: laser coagulation, electrocoagulation, radiofrequency, mucotomy. In most cases, lasercoagulation was chosen.

Results: Nasal permeability in children with chronic hypertrophic rhinitis is altered in most cases. The most frequently affected age groups were 10-14 years with a rate of $61.53 \%$, the majority being from urban living environment in $63.08 \%$. The most representative clinical signs and symptoms according to this study are difficult nasal breathing in $96.93 \%$ and mouth breathing $-89.24 \%$. From the indicated surgical methods, laser coagulation of the nasal cornets was performed in $47.7 \%$ of cases. It proved to be more effective and less invasive compared to electrocoagulation performed in $44.6 \%$ of cases and partial mucotomy in $4.62 \%$.

Conclusions: From the data obtained in the study, we opted for laser coagulation of the lower nasal horns, which has a faster healing period, shorter postoperative complications, greater acceptability from the doctor and patient.
\end{abstract}

Key words: chronic hypertrophic rhinitis, treatment, laser coagulation.

Cite this article

Gheorghian E, Maniuc M, Ababii P, Danilov L. Clinical and treatment aspects in chronic hypertrophic rhinitis in children. Mold Med J. 2021;64(3):11-15. https://doi.org/ 10.52418/moldovan-med-j.64-3.21.02.

\section{Introduction}

Chronic hypertrophic rhinitis is a common problem in childhood and adolescence and has a negative impact on both physical, social and psychological well-being [1]. The prevalence of chronic hypertrophic rhinitis in children (CHRC) is $20-55 \%$, and in the last 5 years this index is increasing. Epidemiological data on chronic hypertrophic rhinitis estimate that more than 200 million people worldwide suffer from it. In the pediatric population, they showed a prevalence of $16.1 \%$ by the age of 5 years and a prevalence of $42.3 \%$ by the age of 14 [2]. The term "chronic rhinitis, hypertrophic" defines a state of congestion of the mucous membrane and underlying tissues of the nasal concha so that it reduces considerably inferior nasal airway with the functional consequence of constant nasal obstruction [3].

Although chronic hypertrophic rhinitis in children (CHRC) is a fairly common nosological entity in children, its symptoms are often subtle and nonspecific and this can lead to overlooking the diagnosis [4]. Usually the diagnosis of chronic hypertrophic rhinitis in children is based on symptoms that evolve for more than three months and some abnormalities determined by endonasal examination or imaging examination [5]. Patients with $\mathrm{RCHC}$ often receive several types of medications, decongestants, oral antibiotic treatments that can change the clinical presentation, especially in the early stages of the disease [6].

The initial management of RCHC is medical, with goals that include reducing inflammation, improving drainage, and eradicating pathogens. Drug treatment is the first line of approach to the treatment of chronic rhinitis. Depending on the type, duration and severity of symptoms, drug treatment in chronic hypertrophic rhinitis may use the following classes of drugs:

- Nasal decongestants - can be used in both topical and oral form;

- H1 anti-receptor antihistamines, used in patients with allergic rhinitis;

- Intranasal or oral corticosteroids;

- Cromone: sodium cromoglycate and nedocromil sodium;

- Anticholinergics: ipratropium bromide;

- Antileukotrienes;

- Specific immunotherapy, used in allergic rhinitis;

- Intramucosal injection of cortisone [7-9]. 
In case of inefficiency of drug treatment, surgical treatment is considered $[8,9]$. Surgical treatment is reserved for cases where the correct and complete medication performed during 3 consecutive months is ineffective or in irreducible hypertrophy of the nasal cones, when surgical treatment is applied as a first-line method [10].

The specialty literature describes multiple surgical techniques to reduce the size of the nasal cones, the choice which depends on several factors: the type of hypertrophy, the experience and habit of the surgeon, the technical equipment of the clinic where the surgery is performed and, last but not least, the costs of intervention [11]. In case of reducible nasal corneal hypertrophy, several techniques are available, such as lateralization of the nasal cornet with or without submucosal vasotomy, mucotomy (partial, total, submucosal, anterior), electrocautery, diathermic coagulation, radiofrequency coagulation, vaporization or / and laser coagulation $[10,11]$.

A simple method of restoring nasal permeability is to lateralize the cornets. The procedure consists of fracturing and lateral mobilization of the lower nasal cornet in a controlled manner using a blunt instrument. The technique is often used in combination with other procedures. The technology is quite safe and easy to use, however in pronounced irreducible hypertrophy, it may not be sufficient [1].

Cauterization of the nasal horns by radiofrequency is another method of treatment of chronic hypertrophic rhinitis. It provides for the use of high frequency energy, with the aim of coagulating the tissues and thus reducing the volume of the cornet and reducing nasal obstruction. The radio frequency is generated by a specially designed device. The electric current is transmitted through an electrode to the tissues producing energy release, evaporation of cell fluid, which results in tissue coagulation and narrowing of the nasal cones.

The maximum effect occurs 7-14 days after the procedure. The technique has a number of advantages, namely: it eliminates the risk of bleeding and thus the need for nasal tamponade, excludes the need for general anesthesia in adults, so the method falls within the requirements of ex officio surgery $[12,13]$.

Microdebrider mucotomy is a beneficial alternative in chronic hypertrophic rhinitis because it has the advantage of reducing the hypertrophied and hyperplastic tissue of the lower cornet under direct visualization, without bone lesions and without deep thermal effect [14].

The mucotomy technique with the microdebrider can be done with local anesthesia, by infiltration, or with general anesthesia, depending on the size of the nasal cornet hypertrophy, the existing comorbidities, but also the patient's wishes. The entire tissue of the hypertrophied cornet is resected and with predilection of the posterior third and the tail of the inferior nasal cornet, which are then aspirated on the aspiration system of the apparatus. The results of cornet surgery with the microdebrider prove to be very good, with favorable long-term functional results $[2,14]$.

Electrocoagulation was the first method recorded for the
RCHC treatment and has constantly evolved in its practice. This method consists in decreasing the size of the inferior nasal cone by cauterization due to the volumetric reduction of the nasal cone due to the retraction following the healing process that follows. The technique can be performed by local anesthesia (adults only) or general, under direct endoscopic visualization and usually requires nasal tamponade [14]. The long-term results of this technique are controversial because the recurrence rate of cornet hypertrophy is high $[6,10]$.

Cornetotomy or partial cornetectomy is performed in advanced hypertrophies of the nasal cornet when hyperplastic changes of connective tissue and turbine bone occur. Cornetotomies are performed submucosally by creating two flaps: medial and lateral, and removing excess connective and bone tissue. It is indicated in the hypertrophy of the head and body of the nasal cornet, less often in the muriform hypertrophy of the cornet tail. If done technically correctly, there is practically no open area of the wound and a significant volumetric reduction of the nasal cone is obtained. The procedure has been applied in several studies, which have shown a lasting positive effect on nasal breathing $[4,11,15]$.

Regarding the extensive resections of nasal cones, it should be mentioned that at present they belong to the field of the past, having a predominantly historical interest, due to various complications - atrophic rhinitis, "empty nose" syndrome, which has generated new scientific research and research to make the surgical approach much more costeffective. Thus, the concept of minimally invasive endonasal surgery was developed, which provides for the maximum preservation of the pituitary mucosa - the noble element of the nasal fossae [15-17]. Minimally invasive surgery involves surgical maneuvers, which are related to the limited ablation of pathological tissues with the maximum possible respect for healthy skin and surrounding tissues. This type of surgery has clearly superior advantages over radical surgery, because the repair processes are evolving much more favorably, in connection with the considerable reduction of surgical trauma.

\section{Material and methods}

The study included 65 patients, aged 5 to 18 years, being hospitalized in the ENT department of the Emilian Cotaga Clinic with the diagnosis of chronic hypertrophic rhinitis, during January 2018 - December 2020. This study was achieved through retrospective analysis of complicated data from clinical observation sheets and documents accompanying the data.

\section{Laser coagulation of the nasal horns}

Several types of lasers are known, but in nasal cornet surgery the most common are the Diode laser, the PotassiumTitanium-Phosphate (KTP) laser and the $\mathrm{CO}_{2}$ laser. In the first two types, the conduction of the laser beam is performed through fiber and direct contact with the tissues of the nasal horns, which is an advantage. Another difference be- 

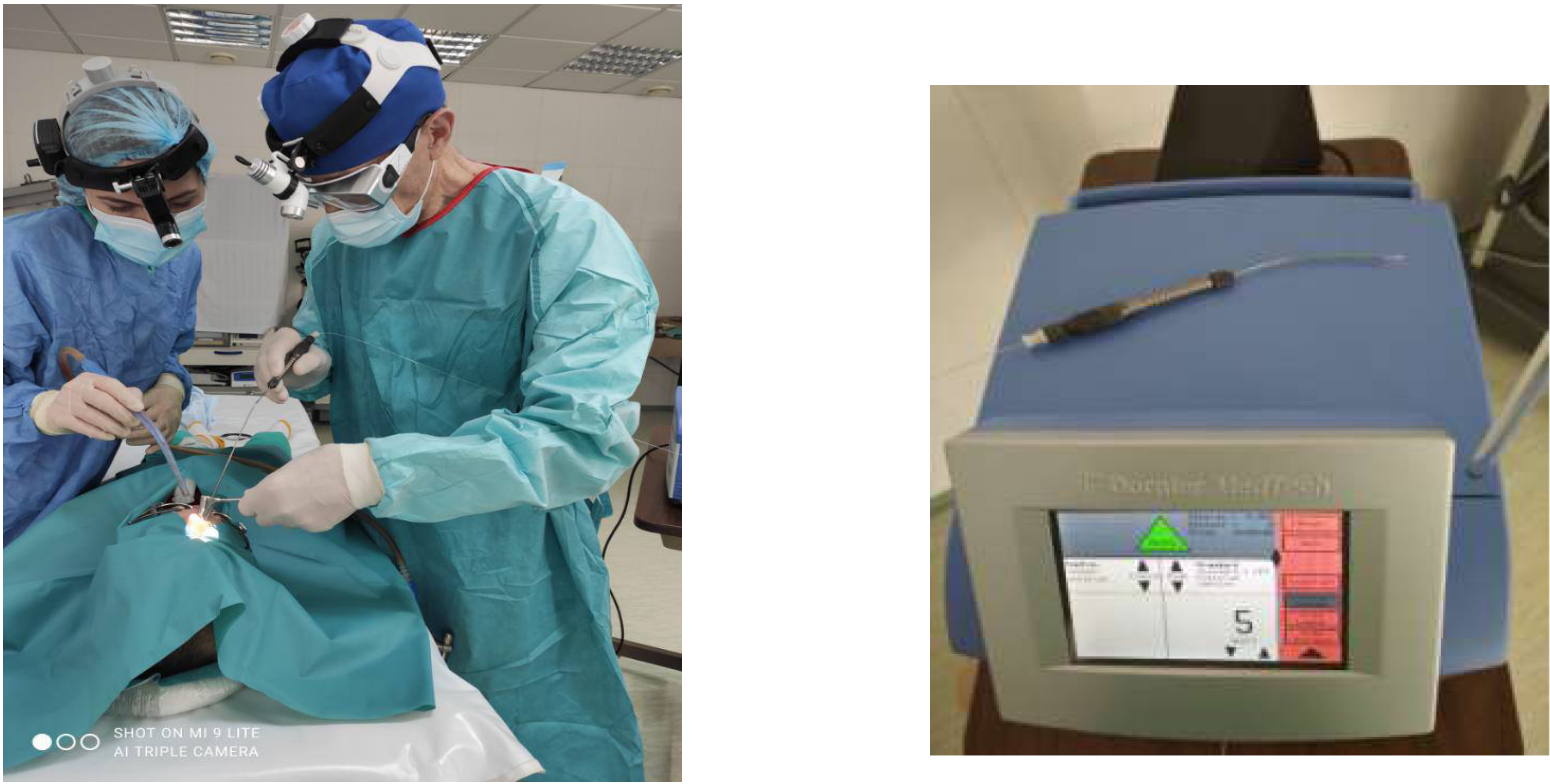

Fig. 1. Diode Laser (Medilas D LiteBeam +)
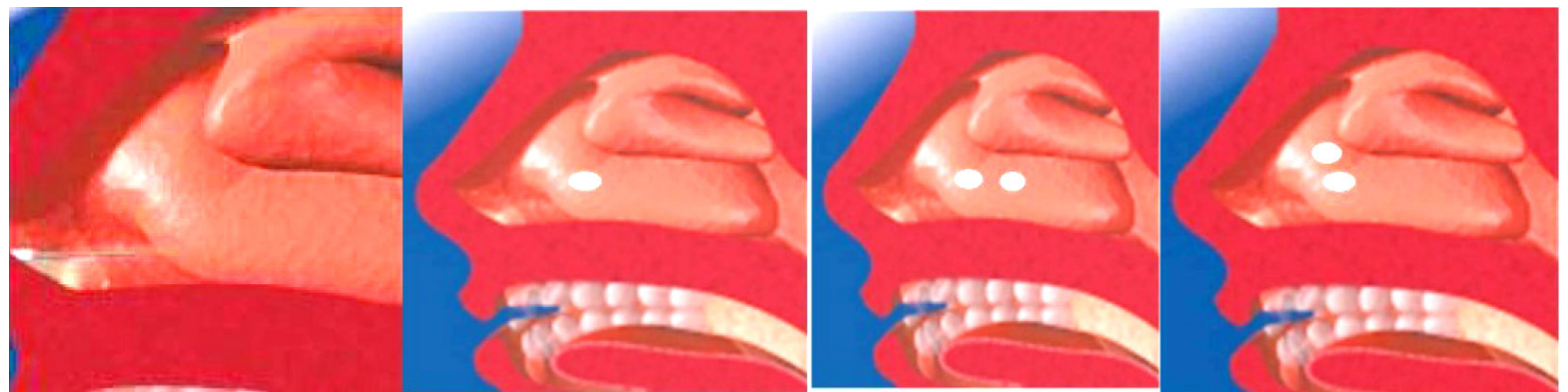

Fig. 2. Laser application techniques in chronic hypertrophic rhinitis in children (CHRC)

tween the first types and the $\mathrm{CO}_{2}$ laser is the wavelength of the emitted light and its ability to penetrate deep. The effect of the wave is strictly on the mucosa of the lower nasal cornet, the laser having no effect on the skeleton.

In the case of diode laser intervention (fig. 1), the volume of the lower nasal cone decreases by the retraction of the loose tissue secondary to the thermal lesions of the tissues. The $\mathrm{CO}_{2}$ laser produces necrotic debris and fibroepithelial reshaping, which replace the vaporized mucosa [18].

The application of choice is the submucosal one, and the important condition is the use of the endoscopic technique (fig. 2). The advantage of this technique is the absence of bleeding of the operative field and the need for nasal tamponade, minimal damage to the mucosal surface of the nasal cone, low reduction of possible disturbance of mucociliary clearance and the possibility of bleeding and scarring in the postoperative period. The operation in adults can be performed under local anesthesia.

\section{Results and discussion}

A number of risk factors were analyzed that could lead to the development of chronic hypertrophic rhinitis and to the worsening of the evolution of the subsequent diagnosis.

One of the main factors is the distribution according to the age of the patients, thus there was a higher addressing of patients aged $10-14$ years with a rate of $61.53 \%$, followed by those aged 5-9 years with a rate of $24.62 \%$, then being followed by those aged $15-18$ with a rate of $13.85 \%$. The results are shown in fig. 5.

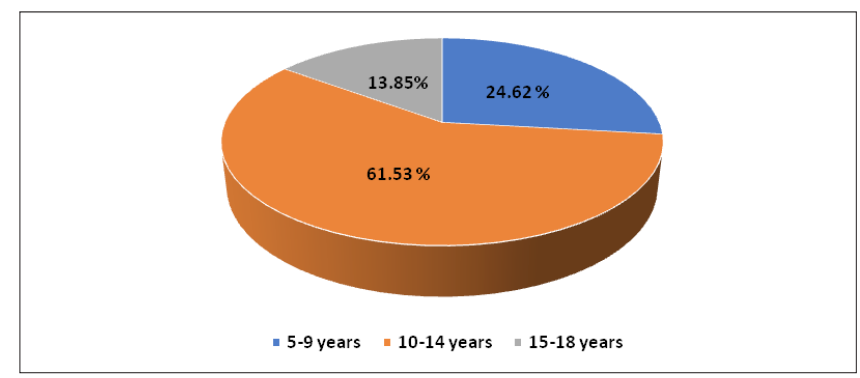

Fig. 5. Distribution of patients by age groups

Among the social factors that can influence the evolution of RCHC is the living environment of patients. Thus, according to figure 6 , the number of patients is higher in the urban living environment -41 cases, with an incidence rate of $63.08 \%$, and in the rural living environment - 24 cases with a rate of $36.92 \%$. 


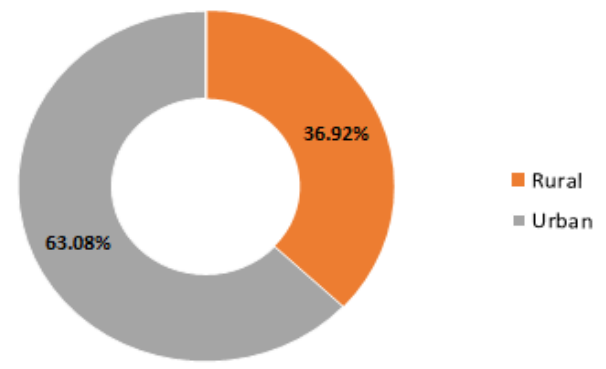

Fig. 6. Distribution of the number of cases depending on the living environment

Based on the data obtained from the study on a group of 65 patients, it is observed according to fig. 7 , the main symptoms were: difficult nasal breathing in $96.93 \%$ and mouth breathing $-89.24 \%$. Other symptoms presented were: nasal voice in $83.08 \%$, fatigue in $78.47 \%$, snoring in $70.77 \%$, sero-mucous secretions in $66.16 \%$, hyposmia in $49.24 \%$ and headache in $32.31 \%$ cases.

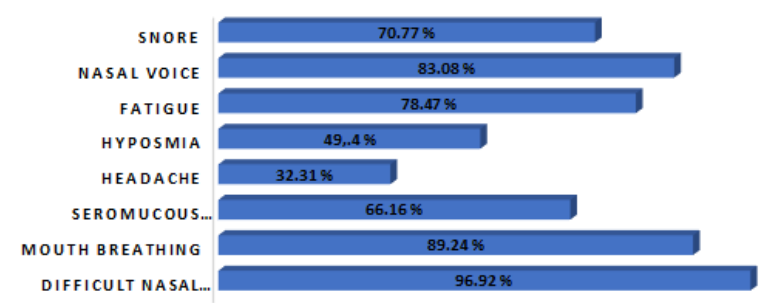

Fig. 7. Distribution of data depending on the symptoms presented

From the multitude of methods listed in chronic hypertrophic rhinitis in children (CHRC) surgery, in 31 cases, laser coagulation with a rate of $47.7 \%$ was chosen. The diode laser was used under general anesthesia, according to the method developed in the clinic, aseptic conditions were observed. Electrocoagulation applied in 29 cases at a rate of $44.62 \%$, and 3 cases $-4.62 \%$ for the partial mucotomy, and in other two cases - $3.06 \%$ another method was chosen (fig. 8).

One of the first studies on laser reduction of hyperplastic lower nasal turbinates were performed by Lenz et al., who treated over 2000 patients with nasal obstruction

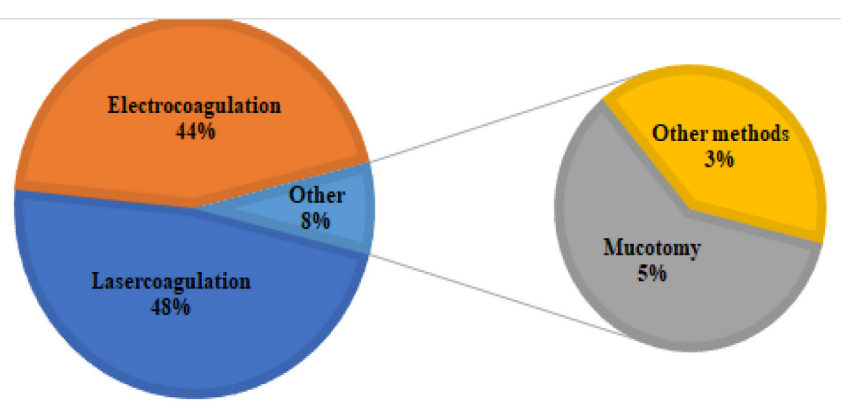

Fig. 8. Distribution of cases depending on the surgical method performed due to vasomotor rhinitis [18]. In $80 \%$ of patients there was an improvement in nasal breathing and a completely free breathing after a period of about 10 years. Similar results were demonstrated by Levine HL. in a trial using laser coagulation in 29 patients suffering from allergic or vasomotor rhinitis [1]. After a monitoring period of $6 \pm 12$ months, $81 \%$ of patients described a subjective improvement in nasal breathing, less nasal congestion [19].

Reduction of hypertrophied nasal cornets by diode laser is safe, minimally invasive and effective in relieving the symptoms associated with chronic hypertrophic rhinitis. To date, there are only a few reports in the literature on mussel treatment. Min et al. treated 53 patients with diode laser vasomotor rhinitis $(810 \mathrm{~nm})$ [5]. The results on nasal obstruction were very satisfactory. In another investigation, 30 patients treated endoscopically with a diode laser (940 nm) were able to achieve an improvement in nasal respiration by $85 \%$ [3]. DeRowe et al. noticed that no more than $41 \%$ of patients received improved postoperative nasal breathing [8].

Hoffmann et al. performed the laser treatment of the lower turbines using the continuous wave diode laser [12]. The laser light was applied through endonasal endoscopes containing a laser channel. A power of $3 \mathrm{~W}$ was established, and the laser tip was slowly pulled into contact from the rear end to the front end of the flow turbine, producing white areas of coagulation ("whitening") of tissue [19]. Additional treatment was performed on hyperplastic tissue in important aerodynamic areas. Seventy-two patients were treated with this method, all noticing an improvement in nasal breathing after six months. Side effects and complications of laser surgery were not described in this study [20]. Compared to most of these treatment modalities, laser surgery of the nasal obstruction is a minimally invasive procedure with a controllable ablation of the hypertrophied tissue causing fewer complications (e.g. , bleeding, dryness, synechiae and pain). The application is easy to make, offering a cost-effective and time-saving method for reducing hyperplastic turbines. Moreover, laser treatment appears to be as effective or even more effective for reducing hyperplastic turbines than most conventional surgical techniques, such as mucotomy, electrocautery, radiofrequency [15]. More invasive (radical) operating methods, such as lower turbinoplasty, submucosal turbinectomy, lateral fracture, and partial and total turbinectomy have the advantage of partially removing large hyperplastic parts of the turbinates, leading to a reduction in obstructive tissue and better results. However, these methods have a higher invasiveness, a longer recovery and much higher costs than laser therapy [4]. Although the total turbinectomy produced a substantial increase in the level of nasal air, it also led to the destruction of the cilia, atrophy of the mucous membrane and chronic nasal infections, with a reduction in the moisturizing capacity and the nasal mucosa.

Submucosal diathermy was first used by Neres FE. [3] in 1907 and developed rapidly in the following years. Beck IC. led the 1930s by the unipolar method [14] and Hurd LM. 
introduced bipolar diathermy in 1931 [20]. The F. target submucosal diathermy is current-induced scarring and obliteration in the submucosa of the lower nasal horns. The results of therapy after submucosal diathermy are evaluated very differently in the literature. The short-term results are good and exceed $80 \%$, with a higher success of therapy in non-allergic patients [9]. However, if the monitoring period is longer, the results of the therapy are clearly worse [2]. A retrospective study showed that after 2 years, only $36 \%$ of patients treated were satisfied with the outcome of treatment [19].

From the data obtained in the study, we opted for laser coagulation of the lower nasal horns, which has a faster healing period, shorter postoperative complications, greater acceptability from the doctor and patient.

\section{Conclusions}

Nasal permeability in children with chronic hypertrophic rhinitis is altered in most cases. The most frequently affected age groups were $10-14$ years with a rate of $61.53 \%$, the majority being from urban living environment $63.08 \%$. The most representative clinical signs and symptoms according to this study are difficult nasal breathing in 96.93\% and mouth breathing in $89.24 \%$.

From the indicated surgical methods, laser coagulation of the nasal cornets was performed in $47.7 \%$ of cases. It proved to be more effective and less invasive compared to electrocoagulation performed in $44.6 \%$ of cases and partial mucotomy in $4.62 \%$.

\section{References}

1. Levine HL. Lasers in endonasal surgery. Otolaryngol Clin North Am. 1997;30(3):451-455.

2. Hellings PW, Klimek L, Cingi C, Agache I, Akdis C, Bachert C, et al. Non-allergic rhinitis: position paper of the European Academy of Allergy and Clinical Immunology. Allergy. 2017;72(11):1657-1665. doi: 10.1111/ all.13200.

3. Neres FE. Voltaic turbinal puncture for the relief of intumescent and hypertrophic rhinitis. J Am Med Ass. 1907;44.

4. Komshian SR, Cohen MB, Brook C, Levi JR. Inferior turbinate hypertrophy: a review of the evolution of management in children. Am J Rhinol Allergy. 2019;33(2):212-219. doi: 10.1177/1945892418815351.

\section{Authors' ORCID iDs and academic degrees}

Emilia Gheorghian, MD, Graduate Student - https://orcid.org/0000-0002-1688-5849

Mihail Maniuc, MD, PhD, Professor of Otorhinolaryngology - https://orcid.org/0000-0002-5340-5779

Polina Ababii, MD, PhD, Assistant Professor of Otorhinolaryngology - https://orcid.org/0000-0002-7206-4906

Lucian Danilov, MD, PhD, Professor of Otorhinolaryngology - https://orcid.org/0000-0002-2834-0395

\section{Authors' contributions}

EG developed the first manuscript; MM interpreted the data and critically revised the manuscript. PA interpreted the statistical data. LD reviewed the clinical data. All authors reviewed the material and approved the final version of the manuscript.

\section{Funding}

This study was supported by Nicolae Testemitanu State University of Medicine and Pharmacy. The research was the initiative of the authors. The authors are independent and take responsibility for the integrity of the data and the accuracy of the data analysis.

\section{Ethics approval and consent to participate}

The study was approved by the Research Ethics Committee of Nicolae Testemitanu State University of Medicine and Pharmacy (protocol No 20.80009.8007.14 of 17.04.2021). Informed consent was obtained from all study participants.

Conflict of Interests. No competing interests were disclosed.
, Kim HS, Yun YS, Kim CS, Jang YJ, Jung TG. Contact laser turquency is a safe and effective treatment of turbinate hypertrophy Laryngoscope. 2001;111(5):894-899. doi: 10.1097/00005537-200105000-00025.

.

A, Landsberg R, Leonov Y, Katzir A, Ophir D. Subjective doi: 10.2500/105065898781390145.

Lippert BM, Werner JA. Treatment of the hypertrophic inferior turbinate. .

(1) Ostryi i hronicheskii rinosinusit: etiologiia, cow; 2014.70 p. Russian.

Larrabee YC, Kacker A. Which inferior turbinate reduction technique 815. doi: 10.1002/lary.24182

12. Hoffmann JF, Parkin JL. Rhinologic applications of laser surgery. Otolaryngol Clin North Am. 1990;23(1):19-28. 2016;127(2):303-308. doi: 10.1002/lary.26264 turbinate. Ann Otol Rhinol Laryngol. 1939;39:349-363.

Fer Findemann J, Scheithauer MO, Hoffmann TK, Veit JA. Nas456. doi: 10.1007/s00106-017-0349-5. German.

Lal D, Corey JP. Acoustic rhinometry and its uses in rhinology and 2004;12(4):397-405. doi: 10.1016/j.fsc.2004.04.002.

, Krempl GA. Radiofrequency treatment of turbinate hyperlaryngol Head Neck Surg. 2004;130(3):291-299. doi: 10.1016/j. otohns.2003.11.003.

( with chronic, difficult-to-treat, non-allergic rhinitis using multiple nasa provocation tests. Pediatr Allergy Immunol. 2019;30(3):296-304. doi:

of the inferior turbinate. Arch Otolaryngol. 1931;13:442. bined with septoplasty: Prospective randomized trial. Laryngoscope. report. Indications and procedure. Laser Med. 1997;13:120. 